\title{
ASYMPTOTIC BEHAVIOR OF ALMOST-ORBITS OF REVERSIBLE SEMIGROUPS OF NON-LIPSCHITZIAN MAPPINGS IN BANACH SPACES
}

\author{
JONG SOO JUNG \\ Department of Mathematics \\ Dong-A University \\ Pusan607-714, KOREA \\ E-mail address' jungjs@seunghak donga ac kr \\ JONG YEOUL PARK \\ Department of Mathematics \\ Pusan National University \\ Pusan 609-735, KOREA \\ JONG SEO PARK \\ Department of Mathematics \\ Graduate School, Dong-A University \\ Pusan 607-714, KOREA
}

(Received February 15, 1994 and in revised form October 25, 1995)

\begin{abstract}
Let $C$ be a nonempty closed convex subset of a uniformly convex Banach space $E$ with a Fréchet differentiable norm, $G$ a right reversible semitopological semigroup, and $\mathcal{S}=\{S(t): t \in G\}$ a continuous representation of $G$ as mappings of asymptotically nonexpansive type of $C$ into itself The weak convergence of an almost-orbit $\{u(t): t \in G\}$ of $\mathcal{S}=\{S(t): t \in G\}$ on $C$ is established. Furthermore, it is shown that if $P$ is the metric projection of $E$ onto set $F(\mathcal{S})$ of all common fixed points of $\mathcal{S}=\{S(t): t \in G\}$, then the strong limit of the net $\{P u(t): t \in G\}$ exists.
\end{abstract}

KEY WORDS AND PHRASES: Almost-orbit, fixed point, reversible semitopological semigroup, semigroup of asymptotically nonexpansive type, uniformly convex Banach space

1991 AMS SUBJECT CLASSIFICATION CODES: 47H20, 47H10, 47H09

\section{INTRODUCTION}

Let $C$ be a nonempty closed convex subset of a real Banach space $E$ and let $S=\{S(t): t \geq 0\}$ be a family of mappings from $C$ into itself such that $S(0)=I, S(t+s)=S(t) S(s)$ for all $t, s \in[0, \infty)$ and $S(t) x$ is continuous in $t \in[0, \infty)$ for each $x \in C$. $S$ is said to be

(a) nonexpansive semigroup on $C$ if $\|S(t) x-S(t) y\| \leq\|x-y\|$ for all $x, y \in C$ and $t \geq 0$,

(b) asymptotically nonexpansive semigroup on $C$ [1] if there is a function $k \cdot[0, \infty) \rightarrow[0, \infty)$ with lim $\sup _{t \rightarrow \infty} k(t) \leq 1$ such that $\|S(t) x-S(t) y\| \leq k(t)\|x-y\|$ for all $x, y \in C$ and $t \geq 0$,

(c) semigroup of asymptotically nonexpansive type on $C$ [1] if for each $x \in C$,

$$
\limsup _{t \rightarrow \infty}\left\{\sup _{y \in C}[\|S(t) x-S(t) y\|-\|x-y\|]\right\} \leq 0 ;
$$

see [2] for mappings of asymptotically nonexpansive type. It is easily seen that (a) $\Rightarrow(b) \Rightarrow$ (c) and that both the inclusions are proper (cf. [1, p. 112]).

In [3], Myadera and Kobayashi introduced the notion of almost-orbits of nonexpansive semigroups on $C$ and provided the weak and strong almost convergences of such an almost-orbit in a uniformly convex Banach space; see also [4] for almost-orbits of nonexpansive mappings. Recently, Tan and Xu [5] extended this notion to semigroups of asymptotic nonexpansive type in Hilbert spaces The case of 
general commutative nonexpansive semigroups in uniformly convex Banach spaces was studied by Takahashi and Park [11]. Oka [6] gave the results for the case of commutative asymptotically nonexpansive semigroups in uniformly convex Banach spaces. In particular, Takahashi and Zhang [7] established the convergences of almost-orbits of noncommutative asymptotically nonexpansive semigroups in the same Banach spaces, see [8] for the case of Hilbert spaces

The purpose of this paper is to generalize their results to the case of noncommutative semigroups of asymptotically nonexpansive type Section 2 is a preliminary part In Section 3, we prove several lemmas which are crucial for our discussion. Main results are given in Section 4 First, we establish the weak convergence (Theorem 1) of an almost-orbit $\{u(t): t \in G\}$ of a semigroup $\mathcal{S}=\{S(t): t \in G\}$ of asymptotically nonexpansive type on $C$ in a uniformly convex Banach space with a Fréchet differentiable norm, where $G$ is a right reversible semitopological semigroup. Next, we show that if $P$ is the metric projection of $E$ onto set $F(\mathcal{S})$ of all common fixed points of $\mathcal{S}=\{S(t): t \in G\}$, then the strong limit of the net $\{P u(t): t \in G\}$ exists (Theorem 2). Our proofs employ the methods of Hirano and Takahashi [9], Ishihara and Takahashi [10], Takahashi and park [11], and Takahashi and Zhang [7,8] The results are generalizations of the corresponding results in [5], [7], [8], [11], [12] and [13].

\section{PRELIMINARIES}

Let $E$ be a real Banach space and let $E^{*}$ be its dual. The value of $f \in E^{*}$ at $x \in E$ will be denoted by $(x, f)$ With each $x \in E$, we associate the set

$$
J(x)=\left\{f \in E^{*}:(x, f)=\|x\|^{2}=\|f\|^{2}\right\} .
$$

Using the Hahn-Banach theorem, it is readily verified that $J(x) \neq \emptyset$ The multivalued mapping $J: E \rightarrow E^{*}$ is called the duality mapping of $E$. Let $U=\{x \in E:\|x\|=1\}$ be the unit sphere of $E$ Then a Banach space $E$ is said to be smooth provided the limit

$$
\lim _{t \rightarrow 0} \frac{\|x+t y\|-\|x\|}{t}
$$

exists for each $x, y \in U$. In this case, the norm of $E$ is said to be Gâteaux differentiable. It is said to be Fréchet differentiable if for each $x \in U$, the limit (2.1) is attained uniformly for $y \in U$ It is also known that if $E$ is smooth, then the duality mapping $J$ is single valued. It is easy to see that the norm of $E$ is Fréchet differentiable if and only if for any bounded set $B \subset E$ and any $x \in E$, $\lim _{t \rightarrow 0}(2 t)^{-1}\left(\|x+t y\|^{2}-\|x\|^{2}\right)=(y, J(x))$ uniformly in $y \in E$; see [14].

A Banach space $E$ is called uniformly convex if the modulus of convexity

$$
\delta(\epsilon)=\inf \left\{1-\frac{1}{2}\|x+y\|:\|x\|,\|y\| \leq 1,\|x-y\| \geq \epsilon\right\}
$$

is positive in its domain of definition $\{\epsilon: 0<\epsilon \leq 2\}$. For the properties of $\delta(\epsilon)$, see [15].

For a subset $D$ of $E, \bar{D}$ denotes the closure of $E, \operatorname{co} D$ the convex hull of $D$, and $\overline{c o} D$ the closed convex hull of $E$, respectively.

Let $G$ be a semitopological semigroup, i.e., $G$ is a semigroup with a Hausdorff topology such that for each $a \in G$ the mappings $g \rightarrow a \cdot g$ and $g \rightarrow g \cdot a$ from $G$ to $G$ are continuous. $G$ is said to be right reversible if any two closed left ideals of $G$ have nonempty intersection. If $G$ is right reversible, $(G, \preceq)$ is a directed system when the binary relation " $\preceq "$ on $G$ is defined by $a \preceq b$ if and only if $\{a\} \cup \overline{G a} \supseteq\{b\} \cup \overline{G b}$.

Let $C$ be a nonempty closed convex subset of a Banach space $E$ and let $G$ be a semitopological semigroup. A family $\mathcal{S}=\{S(t): t \in G\}$ of mappings from $C$ into itself is said to be a (continuous) representation of $G$ on $C$ if $\mathcal{S}$ satisfies the following:

(i) $S(t s) x=S(t) S(s) x$ for all $t, s \in G$ and $x \in C$

(ii) for every $x \in C$, the mappings $s \rightarrow S(s) x$ from $G$ into $C$ is continuous 
DEFINITION 1. A representation $\mathcal{S}=\{S(t): t \in G\}$ of $G$ on $C$ is said to be a semigroup of asymptotically nonexpansive type on $C$ if for each $x \in C$,

$$
\inf _{s \in G} \sup _{s \preceq t} \sup _{y \in C}(\|S(t) x-S(t) y\|-\|x-y\|) \leq 0 .
$$

Let $G$ be right reversible and let $\mathcal{S}=\{S(t): t \in G\}$ be a representation of $G$ on $C$ A function $u: G \rightarrow C$ is called an almost-orbit of $\mathcal{S}=\{S(t): t \in G\}$ if

$$
\lim _{s \in G}\left(\sup _{t \in G}\|u(t s)-S(t) u(s)\|\right)=0 .
$$

$\omega(u)$ denotes the set of all weak limit points of subnets of the net $\{u(t): t \in G\}$, and $F(\mathcal{S})=\bigcap_{t \in G} F(S(t))$ the set of all common fixed points of mappings $S(t), t \in G$ in $C$

\section{LEMMAS}

In this section, we prove several lemmas which are crucial in convergence of almost-orbits.

LEMMA 1. Let $C$ be a nonempty closed convex subset of a uniformly convex Banach space $E$ and let $\mathcal{S}=\{S(t): t \in G\}$ be a semigroup of asymptotically nonexpansive type of a right reversible semitopological semigroup $G$ on $C$. Then $F(\mathcal{S})$ is a closed and convex subset of $C$.

PROOF. The closedness of $F(\mathcal{S})$ is obvious. To show convexity, it is sufficient to show that $z=\frac{x+y}{2} \in F(\mathcal{S})$ for all $x, y \in F(\mathcal{S})$. Let $x, y \in F(\mathcal{S}), x \neq y \quad$ If $\lim _{t \in G} S(t) z=z$, then for any $s \in G$,

$$
S(s) z=\lim _{t \in G} S(s) S(t) z=\lim _{t \in G} S(s t) z=\lim _{t \in G} S(t) z=z,
$$

i.e., $z \in F(\mathcal{S})$. Hence it suffices to prove that $\lim _{t \in G} S(t) z=z$. If not, there exists $\epsilon>0$ such that for any $t \in G$, there is $t^{\prime} \in G$ with $t^{\prime} \succeq t$ and

$$
4\left\|S\left(t^{\prime}\right) z-z\right\|=\left\|2\left(S\left(t^{\prime}\right) z-x\right)-2\left(y-S\left(t^{\prime}\right) z\right)\right\| \geq \epsilon .
$$

Choose $d>0$ so small that

$$
(R+d)\left(1-\delta\left(\frac{\epsilon}{R+d}\right)\right)<R,
$$

where $R=\|x-y\|>0$ and $\delta$ is the modulus of convexity of $E$ Since $\mathcal{S}=\{S(t): t \in G\}$ is asymptotically nonexpansive type on $C$, there is $t_{0} \in G$ such that

$$
\sup _{t_{0} \leq t} \sup _{w \in C}(\|S(t) z-S(t) w\|-\|z-w\|) \leq \frac{d}{2}
$$

Put $u=2\left(S\left(t_{0}^{\prime}\right) z-x\right), v=2\left(y-S\left(t_{0}^{\prime}\right) z\right)$. Then $\|u-v\|=4\left\|S\left(t_{0}^{\prime}\right) z-z\right\| \geq \epsilon$. Furthermore, since $t_{0} \preceq t_{0}^{\prime}$, we have

$$
\begin{aligned}
\|u\| & =2\left\|S\left(t_{0}^{\prime}\right) z-x\right\| \\
& =2\left(\left\|S\left(t_{0}^{\prime}\right) z-S\left(t_{0}^{\prime}\right) x\right\|-\|z-x\|\right)+2\|z-x\| \\
& \leq 2 \sup _{t_{0} \preceq t} \sup _{w \in C}(\|S(t) z-S(t) w\|-\|z-w\|)+\|x-y\|<R+d
\end{aligned}
$$

and

$$
\begin{aligned}
\|v\| & =2\left\|y-S\left(t_{0}^{\prime}\right) z\right\| \\
& =2\left(\left\|S\left(t_{0}^{\prime}\right) z-S\left(t_{0}^{\prime}\right) y\right\|-\|z-y\|\right)+2\|z-y\| \\
& \leq 2 \sup _{t_{0} \preceq t} \sup _{w \in C}(\|S(t) z-S(t) w\|-\|z-w\|)+\|x-y\|<R+d .
\end{aligned}
$$

So we have

$$
\left\|\frac{u+v}{2}\right\| \leq(R+d)\left(1-\delta\left(\frac{\epsilon}{R+d}\right)\right),
$$

and hence 


$$
\|x-y\|=\left\|\frac{u+v}{2}\right\| \leq(R+d)\left(1-\delta\left(\frac{\epsilon}{R-d}\right)\right)<R=\|x-y\|
$$

This is a contraction. Therefore, $\lim _{t \in G} S(t) z=z$, which completes the proof

LEMMA 2. Let $C$ be a nonempty closed convex subset of Banach space $E$ Let $G$ be a right reversible semitopological semigroup and let $\mathcal{S}=\{S(t): t \in G\}$ be a semigroup of asymptotically nonexpansive type on $C$. If $\{u(t): t \in G\}$ and $\{v(t): t \in G\}$ are almost-orbits of $\mathcal{S}=\{S(t): t \in G\}$, then $\lim _{t \in G}\|u(t)-v(t)\|$ exists. In particular, for every $z \in F(\mathcal{S}), \lim _{t \in G}\|u(t)-z\|$ exists

PROOF. Put

$$
\phi(s)=\sup _{t \in G}\|u(t s)-S(t) u(s)\|, \quad \psi(s)=\sup _{t \in G}\|v(t s)-S(t) v(s)\|
$$

for $s \in G \quad$ Then $\lim _{s \in G} \phi(s)=\lim _{s \in G} \psi(s)=0$. Let $\epsilon>0$. Since $\mathcal{S}=\{S(t): t \in G\}$ is of asymptotically nonexpansive type on $C$, there exists $t_{0} \in G$ such that

$$
\sup _{t_{0} \unlhd t} \sup _{w \in C}(\|S(t) u(s)-S(t) w\|-\|u(s)-w\|)<\epsilon
$$

for all $s \in G$. On the other hand, since, for any $s, t \in G$,

$$
\begin{aligned}
\|u(t s)-v(t s)\| & \leq \phi(s)+\psi(s)+(\|S(t) u(s)-S(t) v(s)\|-\|u(s)-v(s)\|)+\|u(s)-v(s)\| \\
& \leq \phi(s)+\psi(s)+\sup _{w \in C}(\|S(t) u(s)-S(t) w\|-\|u(s)-w\|)+\|u(s)-v(s)\|,
\end{aligned}
$$

we have

$$
\begin{gathered}
\inf _{t \in G} \sup _{t \preceq \tau}\|u(\tau)-v(\tau)\| \leq \phi(s)+\psi(s)+\sup _{t_{0} \preceq t} \sup _{w \in C}(\|S(t) u(s)-S(t) w\|-\|u(s)-w\|)+\|u(s)-v(s)\| \\
\leq \phi(s)+\psi(s)+\epsilon+\|u(s)-v(s)\|,
\end{gathered}
$$

and then $\inf _{t \in G} \sup _{t \preceq \tau}\|u(\tau)-v(\tau)\| \leq \sup _{t \in G} \inf _{t \preceq s}\|u(s)-v(s)\| \quad$ Thus $\lim _{t \in G}\|u(t)-v(t)\|$ exists. Let $z \in F(\mathcal{S})$ and put $v(t)=z$. Then $v(t)$ is an almost-orbit and hence $\lim _{t \in G}\|u(t)-z\|$ exists

LEMMA 3. Let $C$ be a nonempty closed convex subset of Banach space $E$. Let $G$ be a right reversible semitopological semigroup and let $\mathcal{S}=\{S(t): t \in G\}$ be a semigroup of asymptotically nonexpansive type on $C$. Let $\{u(t): t \in G\}$ be an almost-orbit of $\mathcal{S}=\{S(t): t \in G\} \quad$ If $F(\mathcal{S}) \neq \emptyset$, then there exists $t_{0} \in G$ such that $\left\{u(t): t \succeq t_{0}\right\}$ is bounded.

PROOF. Let $z \in F(\mathcal{S})$. Then, since $\lim _{t \in G}\|u(t)-z\|$ exists by Lemma 2 , there is $t_{0} \in G$ such that $\left\{\|u(t)-z\|: t \succeq t_{0}\right\}$ is bounded. Hence $\left\{u(t): \succeq t_{0}\right\}$ is bounded.

LEMMA 4. Let $C$ be a nonempty closed convex subset of a uniformly convex Banach space $E$ Let $G$ be a right reversible semitopological semigroup and let $\mathcal{S}=\{S(t): t \in G\}$ be a semigroup of asymptotically nonexpansive type on $C$. Let $\{u(t): t \in G\}$ be an almost-orbit of $\mathcal{S}=\{S(t): t \in G\}$ Suppose that $F(\mathcal{S}) \neq \emptyset$. Let $y \in F(\mathcal{S})$ and $0<\alpha \leq \beta<1$. Then for any $\epsilon>0$, there is $t_{0} \in G$ such that

$$
\|S(t)(\lambda u(s)+(1-\lambda) y)-(\lambda S(t) u(s)+(1-\lambda) y)\|<\epsilon
$$

for all $t, s \succeq t_{0}$ and $\lambda \in[\alpha, \beta]$.

PROOF. By Lemma $2, \lim _{t \in G}\|u(t)-y\|$ exists. Let $\epsilon>0$ and

$$
r=\lim _{t \in G}\|u(t)-y\| \text {. }
$$

If $r=0$, since $\mathcal{S}=\{S(t): t \in G\}$ is of asymptotically nonexpansive type on $C$, there exists $t_{0} \in G$ such that

and

$$
\sup _{t_{0} \preceq t} \sup _{w \in C}(\|S(t)(\lambda u(s)+(1-\lambda) y)-S(t) w\|-\|\lambda u(s)+(1-\lambda) y-w\|)<\frac{\epsilon}{2},
$$

$$
\|u(t)-y\|<\frac{\epsilon}{4}
$$


for $t \succeq t_{0}, 0<\lambda<1$ and $s \in G$. Hence for $s, t \succeq t_{0}, 0<\lambda<1$ and $s \in G$,

$$
\begin{aligned}
& \|S(t)(\lambda u(s)+(1-\lambda) y)-(\lambda S(t) u(s)+(1-\lambda) y)\| \\
& \leq \lambda\|S(t)(\lambda u(s)+(1-\lambda) y)-S(t) u(s)\|+(1-\lambda)\|S(t)(\lambda u(s)+(1-\lambda) y)-y\| \\
& \leq \lambda\left(\sup _{t_{0} \preceq t} \sup _{w \in C}(\|S(t)(\lambda u(s)+(1-\lambda) y)-S(t) w\|-\|\lambda u(s)+(1-\lambda) y-w\|)\right) \\
& \quad+\lambda\|\lambda u(s)+(1-\lambda) y-u(s)\|+(1-\lambda)\left(\sup _{t_{0} \preceq t} \sup _{w \in C}(\| S(t)(\lambda u(s)+(1-\lambda) y)-\right. \\
& \quad \quad S(t) w\|-\| \lambda u(s)+(1-\lambda) y-w \|))+(1-\lambda)\|\lambda u(s)+(1-\lambda) y-y\| \\
& <\lambda \frac{\epsilon}{2}+(1-\lambda) \frac{\epsilon}{2}+2 \lambda(1-\lambda)\|u(s)-y\| \\
& <\frac{\epsilon}{2}+\frac{\epsilon}{2}(\lambda(1-\lambda))<\epsilon .
\end{aligned}
$$

Now, let $r>0$. Then we can choose $d>0$ so small that

$$
(r+d)\left(1-c \delta\left(\frac{\epsilon}{r+d}\right)\right)=r_{0}<r
$$

where $\delta$ is the modulus of convexity of $E$ and

$$
c=\min \{2 \lambda(1-\lambda): \alpha \leq \lambda \leq \beta\} .
$$

Let $a>0$ with $2 a+r_{0}<r$. Then there is $t_{0} \in G$ such that

$$
\begin{gathered}
r-a<\|u(s)-y\| \leq r+\frac{d}{2} \text { for } s \succeq t_{0}, \\
\|S(s) u(t)-u(s t)\|<a \text { for } t \succeq t_{0} \text { and } s \in G, \\
\sup _{t_{0} \leq t} \sup _{w \in C}(\|S(t) z-S(t) w\|-\|z-w\|)<\frac{c}{4} d \text { for } z \in C,
\end{gathered}
$$

and

$$
\sup _{t_{0} \preceq t} \sup _{w \in C}(\|S(t) u(s)-S(t) w\|-\|u(s)-w\|)<\frac{c}{4} d \quad \text { for } \quad s \in G .
$$

Suppose that $\|S(t)(\lambda u(s)+(1-\lambda) y)-(\lambda S(t) u(s)+(1-\lambda) y)\| \geq \epsilon$ for some $s, t \succeq t_{0}$ and $\lambda \in[\alpha, \beta] \quad$ Put $z=\lambda u(s)+(1-\lambda) y, u=(1-\lambda)(S(t) z-y)$ and $v=\lambda(S(t) u(s)-S(t) z) \quad$ Then we have

$$
\begin{aligned}
\|u\| & =(1-\lambda)(\|S(t) z-S(t) y\|-\|z-y\|)+(1-\lambda)\|z-y\| \\
& \leq(1-\lambda) \sup _{t_{0} \leq t} \sup _{w \in C}(\|S(t) z-S(t) w\|-\|z-w\|)+(1-\lambda)\|\lambda u(s)+(1-\lambda) y-y\| \\
& <(1-\lambda) \frac{c}{4} d+\lambda(1-\lambda)\|u(s)-y\| \\
& \leq \lambda(1-\lambda)\left((1-\lambda) \frac{d}{2}+r+\frac{d}{2}\right)<\lambda(1-\lambda)(r+d)
\end{aligned}
$$

and

$$
\begin{aligned}
\|v\| & =\lambda(\|S(t) u(s)-S(t) z\|-\|u(s)-z\|)+\lambda\|u(s)-z\| \\
& \leq \lambda \sup _{t_{0} \supseteq t} \sup _{w \in C}(\|S(t) u(s)-S(t) w\|-\|u(s)-w\|)+\lambda(1-\lambda)\|u(s)-y\| \\
& <\lambda \frac{c}{4} d+\lambda(1-\lambda)\left(r+\frac{d}{2}\right) \\
& \leq \lambda(1-\lambda)\left(\lambda \frac{d}{2}+r+\frac{d}{2}\right)<\lambda(1-\lambda)(r+d) .
\end{aligned}
$$

We also have that

$$
\|u-v\|=\|S(t) z-(\lambda S(t) u(s)+(1-\lambda) y)\| \geq \epsilon
$$


and

$$
\begin{aligned}
\lambda u+(1-\lambda) v & =\lambda(1-\lambda)(S(t) z-y)+(1-\lambda) \lambda(S(t) u(s)-S(t) z) \\
& =\lambda(1-\lambda)(S(t) u(s)-y)
\end{aligned}
$$

By the Lemma in [16], we have

$$
\begin{aligned}
\lambda(1-\lambda)\|S(t) u(s)-y\| & =\|\lambda u+(1-\lambda) v\| \\
& \leq \lambda(1-\lambda)(r+d)\left(1-2 \lambda(1-\lambda) \delta\left(\frac{\epsilon}{r+d}\right)\right) \\
& \leq \lambda(1-\lambda)(r+d)\left(1-c \delta\left(\frac{\epsilon}{r+d}\right)\right)=\lambda(1-\lambda) r_{0}
\end{aligned}
$$

and hence $\|S(t) u(s)-y\| \leq r_{0} \quad$ This implies that

$$
\|u(t s)-y\| \leq\|u(t s)-S(t) u(s)\|+\|S(t) u(s)-y\|<a+r_{0}<r-a .
$$

This contradicts the fact $\|u(s)-y\|>r-a$ for $s \succeq t_{0}$. The proof is complete

LEMMA 5. Let $C$ be a nonempty closed convex subset of a uniformly convex Banach space $E$ Let $G$ be a right reversible semitopological semigroup and let $\mathcal{S}=\{S(t): t \in G\}$ be a semigroup of asymptotically nonexpansive type on $C$. Let $\{u(t): t \in G\}$ be an almost-orbit of $\mathcal{S}=\{S(t): t \in G\}$. Suppose that $F(\mathcal{S}) \neq \emptyset$. Then $\lim _{t \in G}\|\lambda u(t)+(1-\lambda) x-y\|$ exists for every $x, y \in F(\mathcal{S})$

PROOF. Let $\lambda \in(0,1)$ and $x, y \in F(\mathcal{S})$. By (2.2), (2.3), and Lemma 4, for any $\epsilon>0$, there exists $t_{0} \in G$ such that

$$
\begin{gathered}
\|S(t)(\lambda u(s)+(1-\lambda) x)-(\lambda S(t) u(s)+(1-\lambda) x)\| \leq \frac{\epsilon}{3} \text { for } t, s \succeq t_{0}, \\
\sup _{t \in G}\|u(t s)-S(t) u(s)\|<\frac{\epsilon}{3} \text { for } s \succeq t_{0}, \\
\sup _{t_{0} \unlhd t} \sup _{w \in C}(\|S(t)(\lambda u(s)+(1-\lambda) x)-S(t) w\|-\|\lambda u(s)+(1-\lambda) x-w\|)<\frac{\epsilon}{3} \text { for } s \in G .
\end{gathered}
$$

Since

$$
\begin{aligned}
\| \lambda u(t s)+ & (1-\lambda) x-y \| \\
\leq & \lambda\|u(t s)-S(t) u(s)\|+\|\lambda S(t) u(s)+(1-\lambda) x-S(t)(\lambda u(s)+(1-\lambda) x)\| \\
& +\sup _{w \in C}(\|S(t)(\lambda u(s)+(1-\lambda) x)-S(t) w\|-\|\lambda u(s)+(1-\lambda) x-w\|) \\
& +\|\lambda u(s)+(1-\lambda) x-y\| \\
& <\epsilon+\|\lambda u(s)+(1-\lambda) x-y\|
\end{aligned}
$$

for all $t, s \in G$, we have

$$
\begin{aligned}
\inf _{t \in G} \sup _{t \preceq \tau}\|\lambda u(\tau)+(1-\lambda) x-y\| & \leq \sup _{t_{0} \preceq t} \| \lambda(u(t s)+(1-\lambda) x-y \| \\
& \leq \epsilon+\|\lambda u(s)+(1-\lambda) x-y\|
\end{aligned}
$$

for all $s \succeq t_{0}$, and then

$$
\inf _{t \in G} \sup _{t \preceq \tau}\|\lambda u(\tau)+(1-\lambda) x-y\| \leq \sup _{t \in G} \inf _{t \preceq s}\|\lambda u(s)+(1-\lambda) x-y\| .
$$

Thus $\lim _{t \in G}\|\lambda u(t)+(1-\lambda) x-y\|$ exists.

LEMMA 6. Let $C$ be a nonempty closed convex subset of a uniformly convex Banach space $E$ with a Fréchet differentiable norm. Let $G$ be a right reversible semitopological semigroup and let $\mathcal{S}=\{S(t): t \in G\}$ be a semigroup of asymptotically nonexpansive type on $C$. Let $\{u(t): t \in G\}$ be an almost-orbit of $\mathcal{S}=\{S(t): t \in G\}$. Then

is at most a singleton.

$$
F(\mathcal{S}) \cap \bigcap_{s \in G} \overline{c o}\{u(t): t \succeq s\}
$$


PROOF. Note that $\bigcap_{s \in G} \overline{c o}\{u(t): t \succeq s\}=\overline{c o} \omega(u)$, see [17] Let $x, y \in F(\mathcal{S})$ Since $E$ has a Fréchet differentiable norm, there exists an increasing function $\gamma: R^{+} \rightarrow R^{+}$such that $\gamma(t) / t \rightarrow 0$ as $t \rightarrow 0^{+}$, and

$$
\begin{aligned}
\frac{1}{2}\|x-y\|^{2}+(h, J(x-y)) & \leq \frac{1}{2}\|x-y+h\|^{2} \\
& \leq \frac{1}{2}\|x-y\|^{2}+(h, J(x-y))+\gamma(\|h\|)
\end{aligned}
$$

for all $h \in E$. Take $h=\lambda(u(t)-x)$ Then

$$
\begin{aligned}
\frac{1}{2}\|x-y\|^{2}+\lambda & (u(t)-x, J(x-y)) \leq \frac{1}{2}\|\lambda u(t)+(1-\lambda) x-y\|^{2} \\
& \leq \frac{1}{2}\|x-y\|^{2}+\lambda(u(t)-x, J(x-y))+\gamma(\lambda\|u(t)-x\|) .
\end{aligned}
$$

Using Lemma 5, we have

$$
\begin{aligned}
\frac{1}{2}\|x-y\|^{2} & +\lambda \inf _{t \in G} \sup _{t \preceq \tau}(u(\tau)-x, J(x-y)) \\
& \leq \frac{1}{2} \lim _{t \in G}\|\lambda u(t)+(1-\lambda) x-y\|^{2} \\
& \leq \frac{1}{2}\|x-y\|^{2}+\lambda \sup _{t \in G} \inf _{t \preceq \tau}(u(\tau)-x, J(x-y))+\gamma(\lambda M),
\end{aligned}
$$

where $\sup _{t \in G}\|u(t)-x\|=M$. Dividing by $\lambda$ and letting $\lambda \rightarrow 0^{+}$, we have $\lim _{t \in G}(u(t), J(x-y))=r$ exists. Of course $r=(v, J(x-y))$ for all $v \in \omega(u)$ and hence for all $v \in \overline{c o} \omega(u)$ Therefore $(v-w, J(x-y))=0$ for all $v, w \in \overline{c o} \omega(u)$, and it readily follows that $F(\mathcal{S}) \cap \bigcap_{s \in G}\{u(t): t \succeq s\}=$ $F(\mathcal{S}) \cap \overline{c o} \omega(u)$ is at most a singleton.

\section{MAIN RESULTS}

In this section, we study the convergence of an almost-orbit $\{u(t): t \in G\}$ of $\mathcal{S}=\{S(t): t \in G\}$

THEOREM 1. Let $E$ be a uniformly convex Banach space with a Fréchet differentiable norm and let $C$ be a nonempty closed convex subset of $E$ Let $F$ be a subset of $C$ and let $G$ be a right reversible semitopological semigroup. Let $\mathcal{S}=\{S(t): t \in G\}$ be a semigroup of asymptotically nonexpansive type on $C$ and let $\{u(t): t \in G\}$ be an almost-orbit of $\mathcal{S}=\{S(t): t \in G\}$. Assume that

(a) $F \subset F(\mathcal{S})$.

Assume also that

(b) if a subnet $\left\{u\left(t_{\alpha}\right)\right\}$ of the net $\{u(t): t \in G\}$ converges weakly to $z$, then $z \in F$.

Then either (i) $F=\emptyset$ and $\|u(t)\| \rightarrow \infty$ or (ii) $F \neq \emptyset$ and the net $\{u(t): t \in G\}$ converges weakly to some $z \in F(\mathcal{S})$.

PROOF. Suppose that some subnet $\left\{u\left(t_{\alpha}\right)\right\}$ of $\{u(t): t \in G\}$ is bounded. Since $E$ is reflexive, a subnet of $\left\{u\left(t_{\alpha}\right)\right\}$ must converge weakly to an element $z \in E$, which is in $F$ by (b). Thus $F=\emptyset$ implies $\|u(t)\|=\infty$.

If, on the other hand, $F \neq \emptyset$, then by Lemma 3, $\{u(t): t \in G\}$ is bounded. So $\{u(t): t \in G\}$ must contain a subnet $\left\{u\left(t_{\alpha}\right)\right\}$ which converges to some $z \in F$ by (b) Since $F \subset F(\mathcal{S})$ and $z \in \overline{c o}$ $\omega(u)=\bigcap_{s \in G} \overline{c o}\{u(t): t \in G\}$, we have

$$
z \in F \cap \bigcap_{s \in G} \overline{c o}\{u(t): t \succeq s\} \subset F(\mathcal{S}) \cap \bigcap_{s \in G} \overline{c o}\{u(t): t \succeq s\} .
$$

Therefore it follows from Lemma 6 that $\{u(t): t \in G\}$ converges weakly to $z \in F(\mathcal{S})$.

As a direct consequence, we have the following corollary, which is a generalization of a result in [5], [7], [8], [11], [12] and [13]

COROLLARY 1. Let $E$ be a uniformly convex Banach space with a Fréchet differentiable norm and let $C$ be a nonempty closed convex subset of $E$ Let $G$ be a right reversible semitopological 
semigroup and let $\mathcal{S}=\{S(t): t \in G\}$ be a semigroup of asymptotically nonexpansive type on $C$ Suppose that $F(\mathcal{S}) \neq \emptyset$ and let $\{u(t): t \in G\}$ be an almost-orbit of $\mathcal{S}=\{S(t): t \in G\} \quad$ If $\omega(u) \subset F(\mathcal{S})$, then the net $\{u(t): t \in G\}$ converges weakly to some $z \in F(\mathcal{S})$

PROOF. The result follows by putting $F=\omega(u)$ in Theorem 1

The following theorem is also a generalization of [7, Theorem 4]

THEOREM 2. Let $C$ be a nonempty closed convex subset of a uniformly convex Banach space $E$ Let $G$ be a right reversible semitopological semigroup and let $\mathcal{S}=\{S(t): t \in G\}$ be a semigroup of asymptotically nonexpansive type on $C$ Suppose that $F(\mathcal{S}) \neq \emptyset$ and let $\{u(t): t \in G\}$ be an almostorbit of $\mathcal{S}=\{S(t): t \in G\} \quad$ Let $P$ denote the metric projection of $E$ onto $F(\mathcal{S})$ Then the strong limit of the net $\{P u(t): t \in G\}$ exists and $\lim _{t \in G} P u(t)=z_{0}$, where $z_{0}$ is a unique element of $F(\mathcal{S})$ such that

$$
\lim _{t \in G}\left\|u(t)-z_{0}\right\|=\min \left\{\lim _{t \in G}\|u(t)-z\|: z \in F(\mathcal{S})\right\} .
$$

PROOF. Since $F(\mathcal{S}) \neq \emptyset$, we know that $\{u(t): t \in G\}$ is bounded and $\lim _{t \in G}\|u(t)-z\|=g(z)$ exists for each $z \in F(\mathcal{S})$. Let $R=\inf \{g(z): z \in F(\mathcal{S})\}$ and $M=\{u \in F(\mathcal{S}): g(u)=R\} \quad$ Then, since $g(z)$ is convex and continuous on $F(\mathcal{S})$ and $g(z) \rightarrow \infty$ as $\|\dot{z}\| \rightarrow \infty, M$ is a nonempty closed convex bounded subset of $F(\mathcal{S})$. Fix $z_{0} \in M$ with $g\left(z_{0}\right)=R$. Since $P$ is the metric projection of $E$ onto $\mathrm{F}(\mathcal{S})$, we have $\|u(t)-P u(t)\| \leq\|u(t)-y\|$ for all $t \in G$ and $y \in F(\mathcal{S})$,

and hence

$$
\inf _{t \in G} \sup _{t \preceq s}\|u(s)-P u(s)\| \leq R .
$$

Suppose that $\inf _{t \in G} \sup _{t \preceq s}\|u(s)-P u(s)\|<R$. Then we may choose $\epsilon>0$ and $t_{0} \in G$ such that

and

$$
\begin{gathered}
\|u(s)-P u(s)\| \leq R-\epsilon \\
\sup _{t_{0} \preceq t} \sup _{w \in C}(\|S(t) u(s)-S(t) w\|-\|u(t)-w\|)<\frac{\epsilon}{4},
\end{gathered}
$$

for all $s \succeq t_{0}$. Since

$$
\sup _{t \in G}\|u(t s)-S(t) u(s)\|<\frac{\epsilon}{4}
$$

$$
\begin{aligned}
\|u(t s)-P u(s)\| \leq & \|u(t s)-S(t) u(s)\|+\|S(t) u(s)-S(t) P u(s)\| \\
& -\|u(s)-P u(s)\|+\|u(s)-P u(s)\| \\
& \leq \phi(s)+\sup _{w \in C}(\|S(t) u(s)-S(t) w\|-\|u(s)-w\|)+\|u(s)-P u(s)\|
\end{aligned}
$$

for all $s, t \in G$ and $\lim _{s \in G} \phi(s)=0$, where $\phi(s)=\sup _{t \in G}\|u(t s)-S(t) u(s)\|$, we have

$$
\|u(t s)-P u(s)\|<\frac{\epsilon}{4}+\frac{\epsilon}{4}+r-\epsilon=R-\frac{\epsilon}{2}
$$

for $s \succeq t_{0}$ and all $t \in G$. Therefore, we obtain

$$
\lim _{t \in G}\|u(t)-P u(s)\|=\inf _{t \in g} \sup _{t \preceq \tau}\|u(\tau)-P u(s)\| \leq R-\frac{\epsilon}{2}<R .
$$

This is a contradiction. So we conclude that

$$
\inf _{t \in G} \sup _{t \preceq s}\|u(s)-P u(s)\|=R .
$$

Now we claim that $\lim _{t \in G} P u(t)=z_{0}$. If not, then there exists $\epsilon>0$ such that for any $t \in G$, $\left\|P u\left(t^{\prime}\right)-z_{0}\right\| \geq \epsilon$ for some $t^{\prime} \succeq t$. Choose $a>0$ so small that

$$
(R+a)\left(1-\delta\left(\frac{\epsilon}{R+a}\right)\right)=R_{1}<R
$$

where $\delta$ is the modulus of convexity of the norm of $E$ We have $\left\|u\left(t^{\prime}\right)-P u\left(t^{\prime}\right)\right\| \leq R+a$ and $\left\|u\left(t^{\prime}\right)-z_{0}\right\| \leq R+a$ for large enough $t^{\prime}$. Therefore 


$$
\left\|u\left(t^{\prime}\right)-\frac{P u\left(t^{\prime}\right)+z_{0}}{2}\right\| \leq(R+a)\left(1-\delta\left(\frac{\epsilon}{R+a}\right)\right)=R_{1}
$$

Since, by Lemma 1, the point $w_{t^{\prime}}=\frac{P u\left(t^{\prime}\right)+z_{0}}{2}$ belongs to $F(\mathcal{S})$, as in the above,

$$
\left\|u\left(t t^{\prime}\right)-w_{t^{\prime}}\right\| \leq \phi\left(t^{\prime}\right)+\sup _{w \in C}\left(\left\|S(t) u\left(t^{\prime}\right)-S(t) w\right\|-\left\|u\left(t^{\prime}\right)-w\right\|\right)+\left\|u\left(t^{\prime}\right)-w_{t^{\prime}}\right\| .
$$

Since $\lim _{s \in G} \phi(s)=0$, there is $t^{\prime} \in G$ such that

and

$$
\phi\left(t^{\prime}\right)<\frac{R-R_{1}}{4}
$$

$$
\sup _{t^{\prime} \preceq t} \sup _{w \in C}\left(\left\|S(t) u\left(t^{\prime}\right)-S(t) w\right\|-\left\|u\left(t^{\prime}\right)-w\right\|\right)<\frac{R-R_{1}}{4}
$$

and hence

$$
\lim _{t \in G}\left\|u(t)-w_{t^{\prime}}\right\|=\inf _{r \in G} \sup _{t \preceq \tau}\left\|u(\tau)-w_{t^{\prime}}\right\|<\frac{R-R_{1}}{2}+R_{1}=\frac{R+R_{1}}{2}<R .
$$

This contradicts the fact $R=\inf \{g(z): z \in F(\mathcal{S})\} \quad$ Thus we have $\lim _{t \in G} P u(t)=z_{0}$ Consequently, it follows that the element $z_{0} \in F(\mathcal{S})$ with $g\left(z_{0}\right)=\min \{g(z): z \in F(\mathcal{S})\}$ is unique. The proof is complete

By Corollary 1 and Theorem 2, we have the following, which is an improvement of [8, Theorem 3] and [5, Theorem 3.3].

COROLLARY 2. Let $C$ be a nonempty closed convex subset of a real Hilbert space $H$ Let $G$ be a right reversible semitopological semigroup and $\mathcal{S}=\{S(t): t \in G\}$ be a semigroup of asymptotically nonexpansive type on $C$. Suppose that $F(\mathcal{S}) \neq \emptyset$. Let $\{u(t): t \in G\}$ be an almost-orbit of $\mathcal{S}=\{S(t): t \in G\}$. Then $\{u(t): t \in G\}$ converges weakly to some $z \in C$ if and only if $u(h t)-u(t)$ converges weakly to 0 for all $h \in G$. In this case, $z \in F(\mathcal{S})$ and $\lim _{t \in G} P u(t)=z$

PROOF. We need only prove the "if" part. By Corollary 1, it suffices to show that $\omega(u) \subset F(\mathcal{S})$ Let $\left\{u\left(t_{\alpha}\right)\right\}$ be a subnet of $\{u(t): t \in G\}$ converging weakly to $y \in C$ Given $\epsilon>0$ Since $\mathcal{S}$ is of asymptotically nonexpansive type and $\left\{u\left(t_{\alpha}\right)\right\}$ is bounded, there exists $t_{0} \in G$ such that for any $\alpha$,

$$
\sup _{t_{0} \preceq t} \sup _{w \in C}\left(\left\|S(t) u\left(t_{\alpha}\right)-S(t) w\right\|-\left\|u\left(t_{\alpha}\right)-w\right\|\right)<\epsilon .
$$

So we have, for $t \succeq t_{0}$ and any $\alpha$,

$$
\begin{aligned}
& \left\|S(t) u\left(t_{\alpha}\right)-S(t) y\right\|^{2}-\left\|u\left(t_{\alpha}\right)-y\right\|^{2} \\
& \quad=\left(\left\|S(t) u\left(t_{\alpha}\right)-S(t) y\right\|-\left\|u\left(t_{\alpha}\right)-y\right\|\right)\left(\left\|S(t) u\left(t_{\alpha}\right)-S(t) y\right\|+\left\|u\left(t_{\alpha}\right)-y\right\|\right) \\
& \quad \leq \sup _{t_{0} \leq t} \sup _{w \in C}\left(\left\|S(t) u\left(t_{\alpha}\right)-S(t) w\right\|-\left\|u\left(t_{\alpha}\right)-w\right\|\right)\left(\operatorname { s u p } _ { t _ { 0 } \leq t } \operatorname { s u p } _ { w \in C } \left(\left\|S(t) u\left(t_{\alpha}\right)-S(t) w\right\|\right.\right. \\
& \\
& \quad<\epsilon(\epsilon+2 M),
\end{aligned}
$$

where $M=\sup _{\alpha}\left\|u\left(t_{\alpha}\right)-y\right\|$. Let $u \in F(\mathcal{S})$ and $\epsilon^{\prime}=\epsilon(\epsilon+2 M)$. Then we have, for $t \succeq t_{0}$ and all $\alpha$,

$$
\begin{gathered}
-\epsilon^{\prime}<\left\|u\left(t_{\alpha}\right)-y^{2}\right\|-\left\|-S(t) u\left(t_{\alpha}\right) y\right\|^{2} \\
=\left\|u\left(t_{\alpha}\right)-u\right\|^{2}+2\left(u\left(t_{\alpha}\right)-u, u-y\right)+\|u-y\|^{2} \\
\quad-\left\|S(t) u\left(t_{\alpha}\right)-u\right\|^{2}-2\left(S(t) u\left(t_{\alpha}\right)-u, u-S(t) y\right)-\|u-S(t) y\|^{2} \\
=\left\|u\left(t_{\alpha}\right)-u\right\|^{2}-\left\|S(t) u\left(t_{\alpha}\right)-u\right\|^{2}+\|u-y\|^{2}-\|u-S(t) y\|^{2} \\
\quad+2\left(u\left(t_{\alpha}\right)-u, S(t) y-y\right)+2\left(u\left(t_{\alpha}\right)-S(t) u\left(t_{\alpha}\right), u-S(t) y\right) .
\end{gathered}
$$

Since $\{u(t): t \in G\}$ is an almost-orbit of $\mathcal{S}=\{S(t): t \in G\}$ and $u(h s)-u(s)$ converges weakly to 0 for all $h \in G$, it follows that

$$
\lim _{\alpha}\left\|S(t) u\left(t_{\alpha}\right)-u\right\|^{2}=\lim _{\alpha}\left\|u\left(t t_{\alpha}\right)-u\right\|^{2}=\lim _{\alpha}\left\|u\left(t_{\alpha}\right)-u\right\|^{2}
$$


and

Thus we have

$$
u\left(t_{\alpha}\right)-S(t) u\left(t_{\alpha}\right)=u\left(t_{\alpha}\right)-u\left(t t_{\alpha}\right) \rightarrow 0 \text { weakly. }
$$

$$
-\epsilon^{\prime} \leq 2(y-u, S(t) y-y)+\|y-u\|^{2}-\|u-S(t) y\|^{2}=-\|y-S(t) y\|^{2}
$$

for $t \succeq t_{0}$, and hence $\lim \sup _{t \in G}\|S(t) y-y\| \leq \epsilon^{\prime}$. Since $\epsilon^{\prime}$ is arbitrary, we have $\lim _{t \in G} S(t) y=y$ Now, for $s \in G$,

$$
S(s) y=\lim _{t \in G} S(s) S(t) y=\lim _{t \in G} S(s t) y=\lim _{t \in G} S(t) y=y,
$$

i e, $y \in F(\mathcal{S})$ and hence $\omega(u) \subset F(\mathcal{S})$ By Corollary 1 , the net $\{u(t): t \in G\}$ converges weakly to some $z \in F(\mathcal{S})$ On the other hand, since $P$ is the metric projection of $H$ onto $F(\mathcal{S})$, we know that

$$
(u(t)-P u(t), P u(t)-y) \geq 0
$$

for all $y \in F(\mathcal{S})$. So, if $P u(t) \rightarrow u$ by Theorem 2 , we have $(z-u, u-y) \geq 0$ for all $y \in F(\mathcal{S})$ Putting $z=y$, we obtain $-\|z-u\|^{2} \geq 0$ and hence $z=u$.

As a direct consequence, we have the following.

COROLLARY 3. Let $C$ be a nonempty closed convex subset of a real Hilbert space $H$ Let $G$ be a right reversible semitopological semigroup and let $\mathcal{S}=\{S(t): t \in G\}$ be a semigroup of asymptotically nonexpansive type on $C$. Suppose that $F(\mathcal{S}) \neq \emptyset$ Let $\{u(t): t \in G\}$ be an almost-orbit of $\mathcal{S}=\{S(t): t \in G\}$. If $\lim _{t \in G}\|u(h t)-u(t)\|=0$ for all $h \in G$, then the net $\{u(t): t \in G\}$ converges weakly to some $z \in F(\mathcal{S})$.

\section{REFERENCES}

[1] KIRK, W.A. and TORREJÓN, R., Asymptotically nonexpansive semigroups in Banach spaces, Nonlinear Analysis TMA 3 (1979), 111-121.

[2] KIRK, W.A., Fixed point theorems for non-Lipschitzian mappings of asymptotically nonexpansive type, Israel J. Math. 17 (1974), 339-346.

[3] MIYADERA, I. and KOBAYASHI, K., On the asymptotic behavior of almost-orbits of nonlinear contraction semigroups in Banach spaces, Nonlinear Analysis TMA, 6 (1982), 349-365

[4] BRUCK, R.E., A simple proof of the mean ergodic theorem for nonlinear contractions in Banach spaces, Israel J. Math. 32 (1979), 107-116.

[5] TAN, K.K. and XU, H.K., Asymptotic behavior of almost-orbits of nonlinear semigroups of nonLipschitzian mappings in Hilbert spaces, Proc. Amer. Math. Soc. 117 (1993), 385-393

[6] OKA, H., Nonlinear ergodic theorem for commutative semigroups of asymptotically nonexpansive mappings, Nonlinear TMA 18 (1992), 619-635.

[7] TAKAHASHI, W. and ZHANG, PEI-JUN, Asymptotic behavior of almost-orbits of semigroups of Lipschitzian mappings in Banach spaces, Kodai Math. J. 11 (1988), 129-140.

[8] TAKAHASHI, W. and ZHANG, PEI-JUN, Asymptotic behavior of almost-orbits of reversible semigroups of Lipschitzian mappings, J. Math. Anal. Appl. 142 (1989), 242-249.

[9] HIRANO, N. and TAKAHASHI, W., Nonlinear ergodic theorems for an amenable semigroup of nonexpansive mappings in a Banach space, Pacific J. Math. 112 (1984), 333-346

[10] ISHIHARA, H. and TAKAHASHI, W., A nonlinear ergodic theorem for a reversible semigroup of Lipschitzian mappings in a Hilbert space, Proc. Amer. Math. Soc. 104 (1988), 431-436

[11] TAKAHASHI, W. and PARK, J.Y., On the asymptotic behavior of almost-orbits of commutative semigroups in Banach spaces, Nonlinear and Convex Analysis, Marcel Dekker, Inc., New York and Basel (1987), 271-293.

[12] KIUCHI, H. and TAKAHASHI, W., Asymptotic behavior of asymptotically nonexpansive families in Banach spaces, Math. Japonica 38 (1993), 627-632.

[13] LAU, A.T. and TAKAHASHI, W., Weak convergence and non-linear ergodic theorems for reversible semigroup of nonexpansive mappings, Pacific J. Math. 126 (1987), 277-294

[14] DIESTEL, J., Geometry of Banach spaces, selected topics, Lecture Notes in Mathematics, 485, Springer-Verlag, Berlin-Heidelberg, New York, 1975.

[15] GOEBEL, K. and REICH, S., Uniform Convexity, Hyperbolic Geometry, and Nonexpansive Mappings, Marcel Dekker, New York, 1984.

[16] GROETSH, C.W., A note on segmenting Mann iterates, J. Math. Anal. Appl. 40 (1972), 369-372

[17] BRUCK, R.E., On the almost-convergence of iterates of nonexpansive mapping in Hilbert space and the structure of the weak $\omega$-limit set, Israel J. Math. 29 (1978), 1-16. 


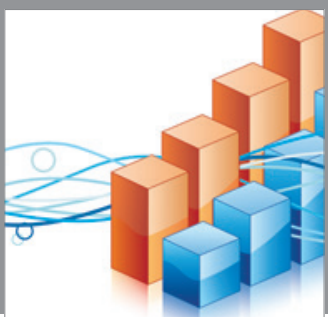

Advances in

Operations Research

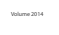

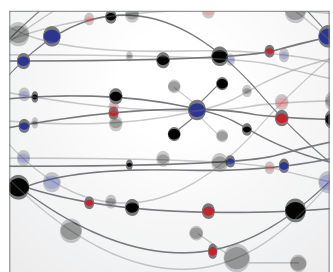

\section{The Scientific} World Journal
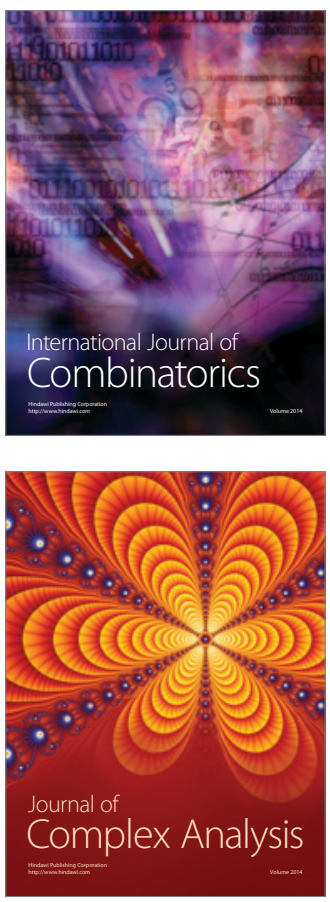

International Journal of

Mathematics and

Mathematical

Sciences
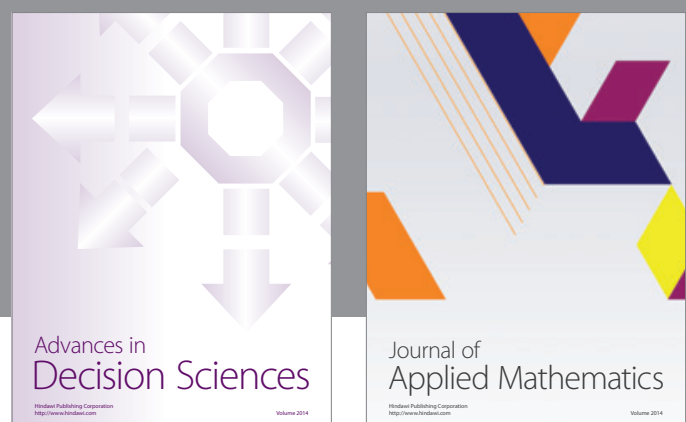

Journal of

Applied Mathematics
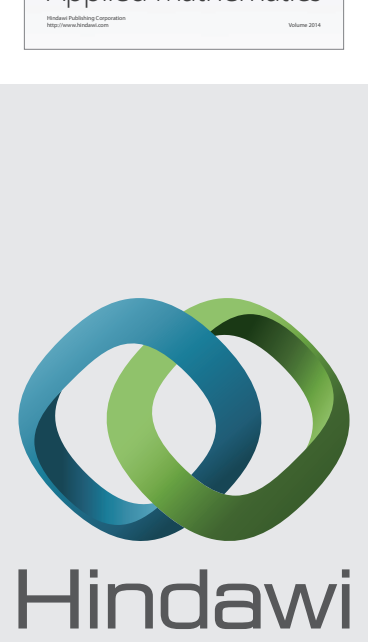

Submit your manuscripts at http://www.hindawi.com
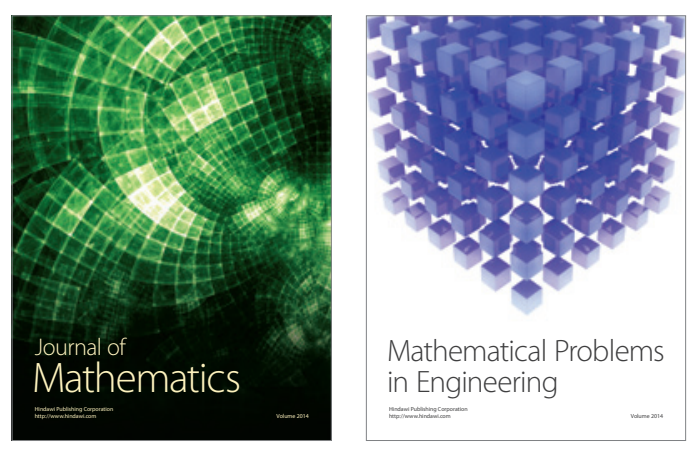

Mathematical Problems in Engineering
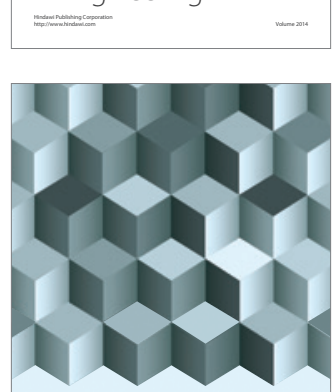

Journal of

Function Spaces
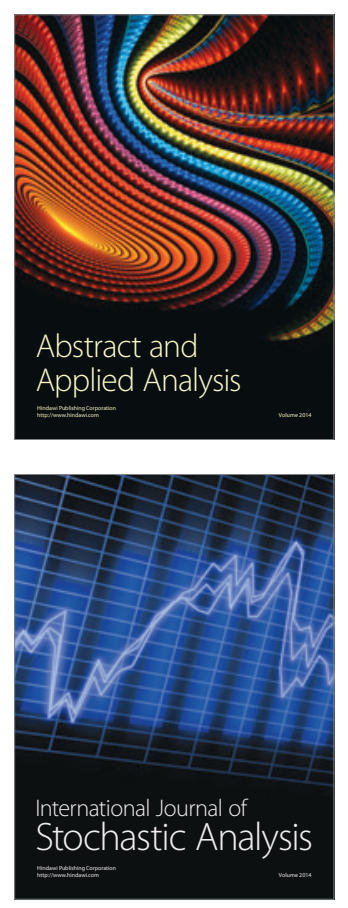

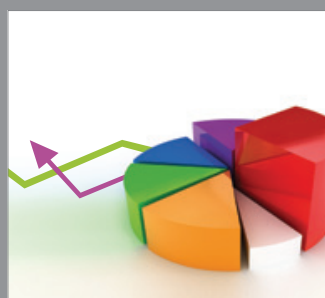

ournal of

Probability and Statistics

Promensencen
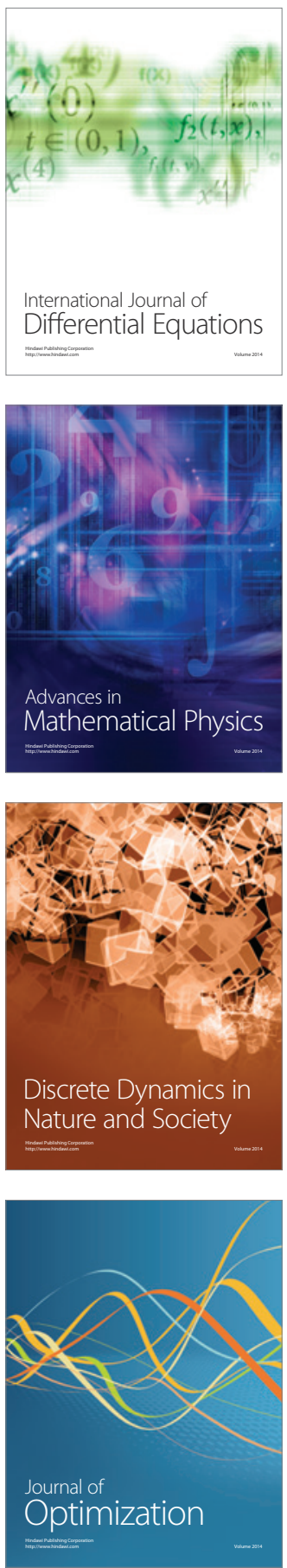